\title{
Diseño Y Aplicación Del Programa De Conservación Auditiva Para La Prevención De Alteraciones De Los Trabajadores Expuestos A Ruido En La Empresa Pública De Hidrocarburos Del Ecuador
}

\author{
Marco Hjalmar Velasco Arellano \\ Carla Sofía Arguello Guadalupe \\ José Franklin Arcos Torres
}

Docente - Investigador de la Facultad de Recursos Naturales ESCUELA SUPERIOR POLITÉCNICA DE CHIMBORAZO - ECUADOR

Patricia del Lourdes Gallegos Murillo

Docente - Investigador de la Facultad de Salud Pública ESCUELA SUPERIOR POLITÉCNICA DE CHIMBORAZO - ECUADOR Andrea Patricia Guapi Auquillas

Investigador externo

doi: 10.19044/esj.2016.v13n2p113 URL:http://dx.doi.org/10.19044/esj.2016.v13n2p113

\begin{abstract}
Shushufindi field is located in the Amazon basin, between the meridians 76 and 77, and the Equinoccial line parallel South. It is oriented in the North-South direction, with approximately $35 \mathrm{Km}$, from long and $7 \mathrm{Km}$ wide, covering an area of about 200 square $\mathrm{km}$. This paper establishes the design and implementation of the programme of hearing conservation for the prevention of disorders of workers occupationally exposed to noise in the field Shushufindi of EPP, developed for workers who are dedicated to activities in the areas of heavy equipment and turbine; establishing the risk factors that may affect health and well-being, allows to implement prevention and protection controls, so workers can carry out their activities in a proper workplace. To carry out the work we performed a literature review and risk factor was identified through the use of a matrix, noise to which workers are exposed, with the use of the method of the three-step (PGV) was carried out the estimation of the risk; determined that the risk of noise value corresponds to 9 considered an intolerable risk, this allowed their preventive management. Hearing conservation program implemented in a systematic way to protect hearing from employees, allows you to prevent the
\end{abstract}


health effects, reduce labor absenteeism, improve industrial productivity, and improve the working conditions of the company.

Keywords: Risk, intolerable and conservation

\section{Resumen}

El campo Shushufindi está ubicado en la Cuenca Amazónica, entre los meridianos 76 y 77, entre la línea Equinoccial y el Paralelo Sur. Está orientado en la dirección norte-sur, con aproximadamente $35 \mathrm{Km}$, de largo y $7 \mathrm{Km}$. de ancho, lo que cubre un área de unos $200 \mathrm{~km}$ cuadrados. El presente trabajo establece el diseño y aplicación del programa de conservación auditiva para la prevención de alteraciones de los trabajadores ocupacionalmente expuestos a ruido en el campo Shushufindi de EPP, desarrollado para los trabajadores que están dedicados a las actividades en las áreas de equipo pesado y turbina; se establece los factores de riesgo que puede afectar a la salud y el bienestar, permite implementar los controles de prevención y protección, por lo tanto los trabajadores pueden desempeñar sus actividades en un centro laboral adecuado. Para realizar la trabajo se realizó una revisión bibliográfica y mediante la utilización de una matriz se identificó el factor de riesgos, ruido al cual los trabajadores están expuestos, con la utilización del método del triple criterio (PGV) se realizó la estimación del riesgo; determinándose que el valor de riesgo de ruido corresponde a 9 considerado un riesgo intolerable, con ello se permite su gestión preventiva. El programa de conservación auditiva implantado en forma sistemática para proteger la audición de los empleados, permite prevenir los efectos en la salud, reducir el ausentismo laboral, mejorar la productividad industrial y mejorar las condiciones laborales de la empresa.

Palabras-claves: Riesgo, intolerable y conservación

\section{Introducción}

Las primeras referencias médicas al ruido proceden del antiguo Egipto, en el siglo XVII AC, y describen, aunque no claramente, el tinnitus. Hipócrates, en el siglo V AC, fue el primero en describir éste como un zumbido ligero y, también, el primero en recomendar que los afectados se mantuvieran lejos de las fuentes de ruido (Dixon, 1982, p. 1772-88).

Pero no fue sino hasta la Revolución Industrial que la comunidad médica en Inglaterra empezó a reconocer que el ruido podía ser un riesgo para la salud. La primera referencia reconocida apareció en la prestigiada revista médica The Lancet entre 1830 y 1831, cuando John Fosbroke afirmó que la sordera de los herreros era consecuencia de su trabajo y que los afectaba de manera gradual, sin que lo notaran (Gilbert, 2004). 
Sin embargo, no hubo ningún médico entre las personalidades incluyendo a Charles Dickens- que, en la época victoriana, pidieron que se legislara para controlar el ruido en las calles de Londres. Esta solicitud condujo a una ley aprobada en 1863, pero no fue sino hasta 1886 que un médico intentó por primera vez evaluar la sordera derivada de las actividades industriales (CDC Office of Health and Safety, 2004).

Thomas Barr, un cirujano que trabajaba en el Hospital del Oído de Glasgow, escuchó el terrible ruido que se hacía al fabricar calderas y afirmó: "Después de tal experiencia, uno se sorprende de que el delicado mecanismo interior de los oídos mantenga su integridad por un solo día que permanezca bajo la acción de tales golpes”. Barr hizo unas grabaciones en la parte más ruidosa de las calderas y logró mostrar el contraste entre el martilleo y la voz humana (Organización Panamericana de la Salud, 2001).

Sin embargo, estos interesantes datos históricos no son tan sorprendentes como nuestra indiferencia a conocer los niveles de ruido a los que estamos expuestos diariamente, a pesar de que actualmente hay una tecnología adecuada y accesible y de que las consecuencias negativas de estar expuestos al ruido en el trabajo, la calle o el hogar, son numerosaps, graves y se han comprobado fuera de toda duda (Martínez, Salamanca, Graficesa, 1969).

Puesto que la capacidad de un ruido para dañar el oído depende fundamentalmente de la cantidad de energía sonora que el trabajador recibe, tan importante es reducir el nivel sonoro, como el tiempo de exposición del mismo, se deberá incluir en su plan de conservación auditiva ( PCA), el mantenimiento preventivo para muchos equipos de trabajo, así como la cantidad de ruido emitido durante su funcionamiento varia de manera importante en función de su estado de mantenimiento (DAIR. Fundación Arauz-Instituto Oto-Rino-Laringológico, 2003).

En la mayoría de los ambientes de planta se emiten ruidos excesivos que comprometen directamente la salud y seguridad de los trabajadores que labora en los puestos de trabajo de turbina y equipo pesado de la Empresa Pública de Hidrocarburos del Ecuador.

En ocasiones también se afecta la comunidad aledaña y la biodiversidad del entorno. Por tales razones, se impone la toma de medidas que mitiguen esta situación, mediante la elaboración e implementación del programa de conservación auditiva para prevenir alteraciones de los trabajadores ocupacionalmente expuestos a ruido laboral.

El presente trabajo tiene como fin la elaboración y aplicación del Programa de Conservación Auditiva para la prevención de alteraciones de los trabajadores ocupacionalmente expuestos a ruido de los departamentos de equipos pesado y turbina de la Empresa Pública de Hidrocarburos del Ecuador del Cantón Shushufindi, basado en la descripción de la empresa en 
su entorno, programa de evaluación y valoración audiométricas , clasificación de las audiometrías, identificación de factores de riesgo.

\section{Metodología}

Este trabajo de investigación se desarrolló en la Empresa Pública de Hidrocarburo del Ecuador en el Cantón Shushufindi provincia de Sucumbíos en los departamentos de Turbina y Equipo pesado durante periodo comprendido entre enero a septiembre del 2012.

o Experimental.- Se realizó un estudio sin manipular deliberadamente las variables independientes. Lo que hicimos en la investigación no experimental es observar los fenómenos tal como se dieron en su contexto natural de la investigación para después analizarlos.

Por los objetivos es aplicada, constructiva o utilitaria: su objetivo es, en cambio, la aplicación, uso y posibles consecuencias de los conocimientos. Si bien depende de los descubrimientos y avances de la investigación básica, busca conocer para actuar, interesa la aplicación sobre la realidad antes que el mero desarrollo de teorías generales.

Por el Lugar Bibliográfica.- Tuvo el propósito de conocer, comparar, ampliar, profundizar, y deducir diferentes enfoques, teorías sobre los diferentes tipos de ruido basándose en documentos bibliográficos cuyos contenidos fundamentaron de manera científica el marco lógico, el debate teórico, también se utilizó en libros revistas, periódicos y otras publicaciones.

Por el lugar es de Campo.- Se realizó el estudio sistemático en la Empresa Pública de Hidrocarburo del Ecuador campo Shushufindi, para tomar contacto en forma directa con la realidad, para obtener información a través de las audiometrías y encuesta de acuerdo con los objetivos del proyecto.

Por el Nivel es descriptiva.- Se especificó los niveles de ruido de los departamentos de turbina y equipo pesado en lo que se refiere a los trabajadores expuesto y de todos los elementos que a partir de la investigación se fueron evidenciando

Nivel explicativo.- Su interés se concentró en buscar el fenómeno que influye en el tipo de hipoacusia o trauma acústico de los trabajadores expuestos de los departamentos de turbina y equipo pesado.

Nivel Propositiva.- Aplicación de un Diseño e Implementación del Programa de Conservación Auditiva para la prevención de alteraciones en los trabajadores ocupacionalmente expuestos a ruido de la Empresa Pública de Hidrocarburo del Ecuador campo Shushufindi.

El método a utilizar en el desarrollo del proyecto de investigación es el científico; puesto que el método científico implica un proceso ordenado y lógico que se sigue para establecer hechos y fenómenos, posibilitando así el 
conocimiento objetivo de la realidad, que contempla el planteamiento de hipótesis, que comprueba las mismas y que explica la realidad de los fenómenos. Para construir y desarrollar la teoría científica que servirá de respaldo en la interpretación de los resultados de nuestra investigación, nos serviremos de los métodos: inductivo y deductivo.

\section{Técnicas e instrumentos para recolección de datos}

Para realizar la investigación de campo en el área de equipo pesado y turbina de la Empresa Pública de Hidrocarburos del Ecuador campo Shushufindi, se empleó técnicas de investigación como: encuestas, entrevista y la observación con sus respectivos instrumentos.

La encuesta: es una técnica destinada a obtener datos de personas cuyas opiniones interpersonales interesan al investigador, en el presente trabajo se aplicó al personal que labora en las áreas de turbina y equipo pesado del campo Shushufindi. El instrumento a utilizar es el cuestionario. Fue estratificado por sectores.

La Observación: Técnica que permite acumular gran parte de datos que constituye la base fundamental para el desarrollo de la investigación proporcionando de una forma clara los factores de riesgo a que están expuestos los trabajadores del área de equipo pesado y turbina de la empresa. El instrumento utilizado es la matriz de riesgo de triple criterio, check-list del ruido industrial, audiometrías, sonometrías, historia clínica ocupacionales.

La entrevista: es una técnica que genera datos por medio de una conversación entre el entrevistador "investigador" y el entrevistado que en este caso fue el señor Coordinador del área de seguridad industrial responsable del campo Shushufindi. Esta técnica se empleó con el fin de obtener información de la autoridad entrevistada. El instrumento utilizado fue el cuestionario de la entrevista.

\section{Resultados}

\section{Población Y Muestra}

Para este estudio se consideró a todo el personal que labora en el área de equipo pesado y turbina de la Empresa Pública de Hidrocarburos del Ecuador, es decir, 22 personas.

Cuadro 1: Grupos de personas que forman parte de la investigación

\begin{tabular}{|c|c|}
\hline ÁREA & POBLACIÓN \\
\hline Equipo pesado & 9 \\
\hline Turbina & 13 \\
\hline Total & 22 \\
\hline
\end{tabular}

Fuente: Empresa Pública de Hidrocarburos del Ecuador Cantón Shushufindi

Elaborado por: Msc. Marco Velasco 
El procedimiento para la recolección de la información fue de la siguiente manera:

Elaboración, validación y reproducción de los instrumentos de recolección de la información.

Aplicación y distribución de la encuesta al personal del área de turbina y equipo pesado.

Explicación de las actividades a desarrollar por los empleados al momento de llenar los cuestionarios para que las respuestas sean contestadas en forma adecuada.

Recolección total de los cuestionarios de encuesta aplicados.

Tabulación y representación en cuadros y gráficos estadísticos.

Como procedimiento para el análisis de resultados se harán uso de las Técnicas Estadísticas y en nuestro caso emplearemos el "t-student" como fuente de demostración de las hipótesis planteadas en este trabajo de investigación.

Cuadro 2. Comparación de los resultados de la encuestas antes y después de la implementación del programa de conservación auditiva en el campo shushufindi.

\begin{tabular}{|c|c|c|c|c|}
\hline \multicolumn{5}{|c|}{ TRABAJADORES EQUIPO PESADO } \\
\hline PREGUNTA & ALTERNATIVA & $\begin{array}{l}\text { PORCENTAJE } \\
\text { ANTES }\end{array}$ & $\begin{array}{l}\text { PORCENTAJE } \\
\text { DESPUÉS }\end{array}$ & OBSERVACIÓN \\
\hline \multirow{5}{*}{$\begin{array}{l}\text { 1. ¿Al trabajador le molesta } \\
\text { el ruido en su puesto de } \\
\text { trabajo? }\end{array}$} & Mucho & 100,00 & 0,00 & \multirow[t]{5}{*}{ MEJORA } \\
\hline & Bastante & 0,00 & 0 & \\
\hline & Regular & 0,00 & 11.89 & \\
\hline & Poco & 0,00 & 88,89 & \\
\hline & Nada & 0,00 & 0,00 & \\
\hline \multirow{5}{*}{$\begin{array}{l}\text { 2. ¿Cuánto tiempo, a lo largo } \\
\text { de su jornada laboral, el } \\
\text { trabajador considera que el } \\
\text { ruido es más molesto? }\end{array}$} & Siempre & 0,00 & 0,00 & \multirow[t]{5}{*}{ MEJORA } \\
\hline & $\begin{array}{l}\text { Más de la Media } \\
\text { Jornada }\end{array}$ & 100,00 & 0,00 & \\
\hline & $\begin{array}{l}\text { Entre la media y } \\
\text { la cuarta parte de } \\
\text { la jornada }\end{array}$ & 0,00 & 0,00 & \\
\hline & $\begin{array}{c}\text { Menos de la } \\
\text { cuarta parte de la } \\
\text { jornada }\end{array}$ & 0,00 & 100,00 & \\
\hline & Nunca & 0,00 & 0,00 & \\
\hline \multirow{4}{*}{$\begin{array}{l}\text { 3. ¿Señale las fuentes de } \\
\text { ruido que le resulten más } \\
\text { molestas al trabajador? }\end{array}$} & Ruido Exterior & 0,00 & 0,00 & \multirow[t]{4}{*}{ IGUAL } \\
\hline & $\begin{array}{c}\text { Ruido } \\
\text { procedente de las } \\
\text { personas } \\
\end{array}$ & 0,00 & 0,00 & \\
\hline & $\begin{array}{l}\text { Ruido de las } \\
\text { Instalaciones }\end{array}$ & 66,67 & 66,67 & \\
\hline & $\begin{array}{l}\text { Ruido de los } \\
\text { equipos de } \\
\text { trabajo }\end{array}$ & 33,33 & 33,33 & \\
\hline \multirow{4}{*}{$\begin{array}{l}\text { 4. ¿El ruido existente } \\
\text { constituye un factor de } \\
\text { distracción importante en el } \\
\text { desarrollo de las tareas? }\end{array}$} & Mucho & 55.56 & 0,00 & \multirow[t]{4}{*}{ MEJORA } \\
\hline & Bastante & 44.44 & 0,00 & \\
\hline & Regular & 0,00 & 22,22 & \\
\hline & Poco & 0,00 & 77,78 & \\
\hline
\end{tabular}




\begin{tabular}{|c|c|c|c|c|}
\hline & Nada & 0,00 & 0,00 & \\
\hline \multirow{5}{*}{$\begin{array}{l}\text { 5. ¿El ruido le dificulta la } \\
\text { concentración mental } \\
\text { requerida en las tareas? }\end{array}$} & Mucho & 66.67 & 0,00 & \multirow[t]{5}{*}{ MEJORA } \\
\hline & Bastante & 33.33 & 0,00 & \\
\hline & Regular & 0,00 & 0,00 & \\
\hline & Poco & 0,00 & 100,00 & \\
\hline & Nada & 0,00 & 0,00 & \\
\hline \multirow{5}{*}{$\begin{array}{l}\text { 6. ¿Es necesario elevar el tono } \\
\text { de voz para hacerse entender } \\
\text { en el desarrollo de su trabajo? }\end{array}$} & Mucho & 88.89 & 0,00 & \multirow[t]{5}{*}{ MEJORA } \\
\hline & Bastante & 11.11 & 0,00 & \\
\hline & Regular & 0,00 & 44,44 & \\
\hline & Poco & 0,00 & 55,56 & \\
\hline & Nada & 0,00 & 0,00 & \\
\hline \multirow{5}{*}{$\begin{array}{l}\text { 7. ¿Es necesario forzar la } \\
\text { atención del receptor a la } \\
\text { distancia habitual de trabajo } \\
\text { para que resulte inteligible } \\
\text { una conversación mantenida } \\
\text { con un tono cómodo para el } \\
\text { emisor? }\end{array}$} & Mucho & 100,00 & 0,00 & \multirow[t]{5}{*}{ MEJORA } \\
\hline & Bastante & 0,00 & 0,00 & \\
\hline & Regular & 0,00 & 0,00 & \\
\hline & Poco & 0,00 & 100,00 & \\
\hline & Nada & 0,00 & 0,00 & \\
\hline \multirow{5}{*}{$\begin{array}{l}\text { 8. ¿Los niveles de ruido } \\
\text { impiden señales acústicas } \\
\text { relevantes o entender } \\
\text { mensajes por megafonía? }\end{array}$} & Mucho & 100,00 & 0,00 & \multirow[t]{5}{*}{ MEJORA } \\
\hline & Bastante & 0,00 & 0,00 & \\
\hline & Regular & 0,00 & 0,00 & \\
\hline & Poco & 0,00 & 100,00 & \\
\hline & Nada & 0,00 & 0,00 & \\
\hline \multirow{2}{*}{$\begin{array}{c}9 . \text { ¿Usted fuma o toma } \\
\text { bebidas alcohólicas fuera de } \\
\text { su jornada laboral? }\end{array}$} & $\mathrm{Si}$ & 33.33 & 11.11 & \multirow[t]{2}{*}{ MEJORA } \\
\hline & no & 66.67 & 88.89 & \\
\hline \multicolumn{5}{|c|}{ TRABAJADORES DE TURBINA } \\
\hline PREGUNTA & ALTERNATIVA & $\begin{array}{l}\text { PORCENTAJE } \\
\text { ANTES }\end{array}$ & $\begin{array}{l}\text { PORCENTAJE } \\
\text { DESPUÉS }\end{array}$ & OBSERVACIÓN \\
\hline \multirow{5}{*}{ 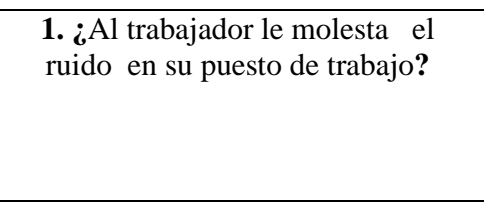 } & Mucho & $100 \%$ & 23.08 & \multirow[t]{5}{*}{ MEJORA } \\
\hline & Bastante & 0,00 & 76.92 & \\
\hline & Regular & 0,00 & 0,00 & \\
\hline & Poco & 0,00 & 0,00 & \\
\hline & Nada & 0,00 & 0,00 & \\
\hline \multirow{5}{*}{$\begin{array}{l}\text { 2. ¿Cuánto tiempo, a lo largo de su } \\
\text { jornada laboral, el trabajador } \\
\text { considera que el ruido es más } \\
\text { molesto? }\end{array}$} & Siempre & 0,00 & 0,00 & \multirow[t]{5}{*}{ MEJORA } \\
\hline & $\begin{array}{l}\text { Más de la Media } \\
\text { Jornada }\end{array}$ & 100,00 & 0,00 & \\
\hline & $\begin{array}{l}\text { Entre la media y } \\
\text { la cuarta parte de } \\
\text { la jornada }\end{array}$ & 0,00 & 0,00 & \\
\hline & $\begin{array}{c}\text { Menos de la } \\
\text { cuarta parte de la } \\
\text { jornada }\end{array}$ & 0,00 & 100,00 & \\
\hline & Nunca & 0.00 & 0,00 & \\
\hline $\begin{array}{l}\text { 3. ¿Señale las fuentes de ruido que } \\
\text { le resulten más molestas al }\end{array}$ & Ruido Exterior & 0.00 & 0,00 & IGUAL \\
\hline
\end{tabular}




\begin{tabular}{|c|c|c|c|c|}
\hline \multirow[t]{3}{*}{ trabajador? } & $\begin{array}{c}\text { Ruido } \\
\text { procedente de las } \\
\text { personas }\end{array}$ & 0.00 & 0,00 & \\
\hline & $\begin{array}{l}\text { Ruido de las } \\
\text { Instalaciones }\end{array}$ & 69,23 & 69,23 & \\
\hline & $\begin{array}{l}\text { Ruido de los } \\
\text { equipos de } \\
\text { trabajo }\end{array}$ & 30,77 & 30,77 & \\
\hline \multirow{5}{*}{$\begin{array}{l}\text { 4. ¿El ruido existente constituye un } \\
\text { factor de distracción importante en } \\
\text { el desarrollo de las tareas? }\end{array}$} & Mucho & 30.77 & 0,00 & \multirow[t]{5}{*}{ MEJORA } \\
\hline & Bastante & 69.23 & 0,00 & \\
\hline & Regular & 0,00 & 23,08 & \\
\hline & Poco & 0,00 & 76,92 & \\
\hline & Nada & 0,00 & 0,00 & \\
\hline \multirow{5}{*}{$\begin{array}{c}\text { 5. ¿El ruido le dificulta la } \\
\text { concentración mental requerida en } \\
\text { las tareas? }\end{array}$} & Mucho & 76.92 & 0,00 & \multirow[t]{5}{*}{ MEJORA } \\
\hline & Bastante & 23.08 & 0,00 & \\
\hline & Regular & 0,00 & 7.69 & \\
\hline & Poco & 0,00 & 92,31 & \\
\hline & Nada & 0,00 & 0,00 & \\
\hline \multirow{5}{*}{$\begin{array}{l}\text { 6. ¿Es necesario elevar el tono de } \\
\text { voz para hacerse entender en el } \\
\text { desarrollo de su trabajo? }\end{array}$} & Mucho & 92.31 & 0,00 & \multirow[t]{5}{*}{ MEJORA } \\
\hline & Bastante & 7.69 & 0,00 & \\
\hline & Regular & 0,00 & 15,38 & \\
\hline & Poco & 0,00 & 84,62 & \\
\hline & Nada & 0,00 & 0,00 & \\
\hline \multirow{5}{*}{$\begin{array}{l}\text { 7. ¿Es necesario forzar la atención } \\
\text { del receptor a la distancia habitual de } \\
\text { trabajo para que resulte inteligible } \\
\text { una conversación mantenida con un } \\
\text { tono cómodo para el emisor? }\end{array}$} & Mucho & 100,00 & 0,00 & \multirow[t]{5}{*}{ MEJORA } \\
\hline & Bastante & 0,00 & 0,00 & \\
\hline & Regular & 0,00 & 0,00 & \\
\hline & Poco & 0,00 & 100,00 & \\
\hline & Nada & 0,00 & 0,00 & \\
\hline \multirow{5}{*}{$\begin{array}{l}\text { 8. ¿Los niveles de ruido impiden } \\
\text { señales acústicas relevantes o } \\
\text { entender mensajes por megafonía? }\end{array}$} & Mucho & 100,00 & 0,00 & \multirow[t]{5}{*}{ MEJORA } \\
\hline & Bastante & 0,00 & 0.00 & \\
\hline & Regular & 0,00 & 0,00 & \\
\hline & Poco & 0,00 & 92,31 & \\
\hline & Nada & 0,00 & 7,69 & \\
\hline \multirow{2}{*}{$\begin{array}{l}\text { 9. ¿Usted fuma o toma bebidas } \\
\text { alcohólicas fuera de su jornada } \\
\text { laboral? }\end{array}$} & $\mathrm{Si}$ & 76.92 & 18,38 & \multirow[t]{2}{*}{ MEJORA } \\
\hline & No & 23.05 & 84,61 & \\
\hline
\end{tabular}

Fuente: Encuesta a los trabajadores de la Empresa EPP-Campo Shushufindi

Elaborado por: Marco Velasco

\section{Demostración de la Hipótesis $\mathbf{N}^{0} 1$}

La vigilancia del ruido en el programa de Conservación Auditiva para la prevención de alteraciones de los trabajadores ocupacionalmente expuestos a ruido de los departamentos de equipos pesado y turbina de la Empresa Pública de Hidrocarburos del Ecuador del Cantón Shushufindi brindan al trabajador un ambiente laboral sano, seguro y productivo. 
Figura 1. Prueba de Hipótesis 1

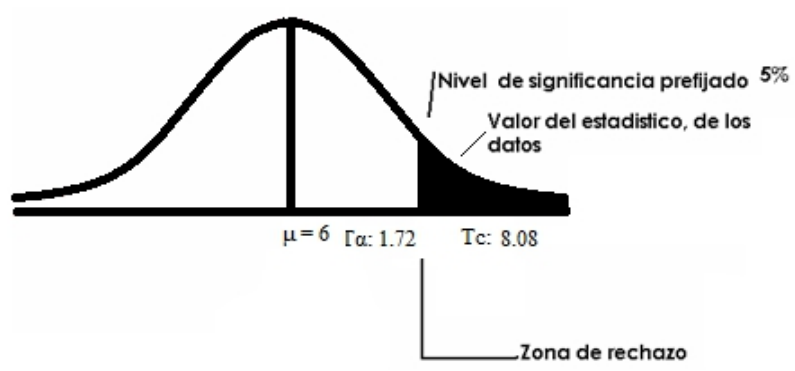

Fuente: Encuestas

Elaborado por: Msc. Marco Velasco

\section{Interpretación}

El estadístico calculado $t_{c}=8.087$ cae en la región de rechazo (se rechaza la hipótesis nula en favor de la hipótesis alternativa) por lo tanto existe suficiente evidencia estadística para afirmar que la vigilancia del ruido en el programa de Conservación Auditiva para la prevención de alteraciones de los trabajadores ocupacionalmente expuestos a ruido de los departamentos de equipos pesado y turbina de la Empresa Pública de Hidrocarburos del Ecuador del Cantón Shushufindi brindan al trabajador un ambiente laboral sano, seguro y productivo.

\section{Demostración de la Hipótesis $\mathbf{N}^{\circ} 2$}

Los controles técnicos en el programa de Conservación Auditiva para la prevención de alteraciones de los trabajadores ocupacionalmente expuestos a ruido de los departamentos de equipos pesado y turbina de la Empresa Pública de Hidrocarburos del Ecuador del Cantón Shushufindi brindan al trabajador un ambiente laboral sano, seguro y productivo.

Figura 2. Prueba de Hipótesis 2

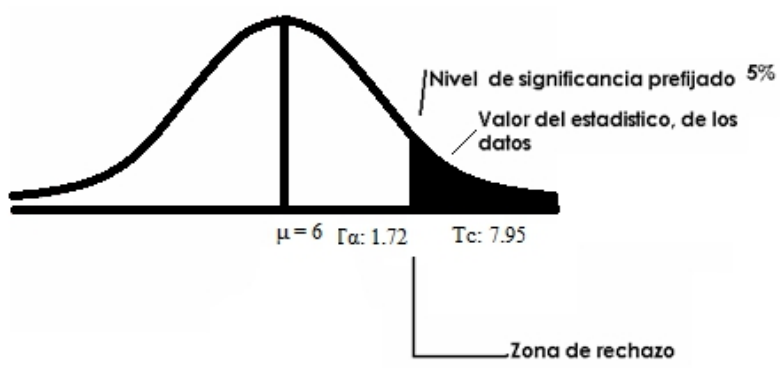

Fuente: Encuesta

Elaborado por: Msc. Marco Velasco 


\section{Interpretación}

El estadístico calculado $t_{c}=7.95$ cae en la región de rechazo (se rechaza la hipótesis nula en favor de la hipótesis alternativa) por lo tanto existe suficiente evidencia estadística para afirmar: Los controles técnicos en el programa de Conservación Auditiva para la prevención de alteraciones de los trabajadores ocupacionalmente expuestos a ruido de los departamentos de equipos pesado y turbina de la Empresa Pública de Hidrocarburos del Ecuador del Cantón Shushufindi brindan al trabajador un ambiente laboral sano.

\section{Demostración de la Hipótesis $\mathbf{N}^{0} 3$}

La educación del trabajador en el programa de Conservación Auditiva para la prevención de alteraciones de los trabajadores ocupacionalmente expuestos a ruido de los departamentos de equipos pesado y turbina de la Empresa Pública de Hidrocarburos del Ecuador del Cantón Shushufindi brindan al trabajador un ambiente laboral sano.

Figura 3. Prueba de Hipótesis 3

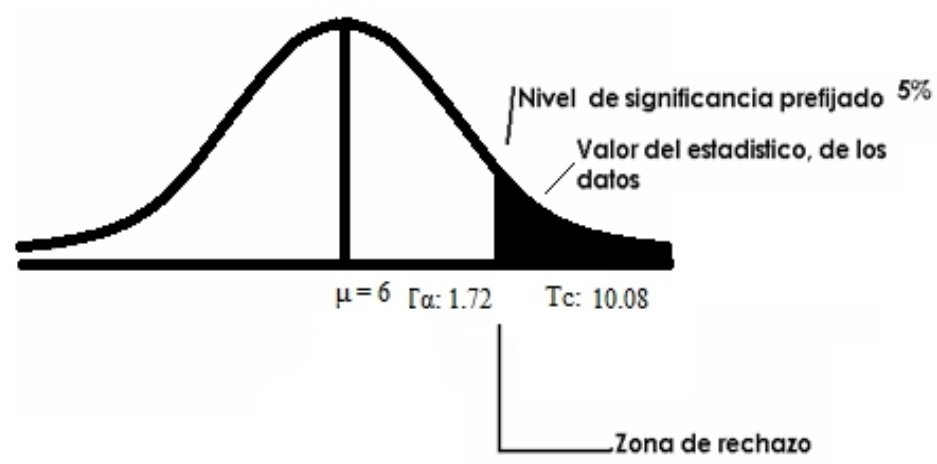

Fuente: Encuesta

Elaborado por: Msc. Marco Velasco

\section{Interpretación}

El estadístico calculado $t_{c}=10.089$ cae en la región de rechazo (se rechaza la hipótesis nula en favor de la hipótesis alternativa) por lo tanto existe suficiente evidencia estadística para afirmar: La educación del trabajador en el programa de Conservación Auditiva para la prevención de alteraciones de los trabajadores ocupacionalmente expuestos a ruido de los departamentos de equipos pesado y turbina de la Empresa Pública de Hidrocarburos del Ecuador del Cantón Shushufindi brindan al trabajador un ambiente laboral sano. 


\section{Demostración de la Hipótesis $\mathbf{N}^{0} \mathbf{4}$}

La selección y uso de aditamentos de protección aditiva (APA) en el programa de Conservación Auditiva para la prevención de alteraciones de los trabajadores ocupacionalmente expuestos a ruido de los departamentos de equipos pesado y turbina de la Empresa Pública de Hidrocarburos del Ecuador del Cantón Shushufindi brindan al trabajador un ambiente laboral sano.

Figura 4. Prueba de Hipótesis 4

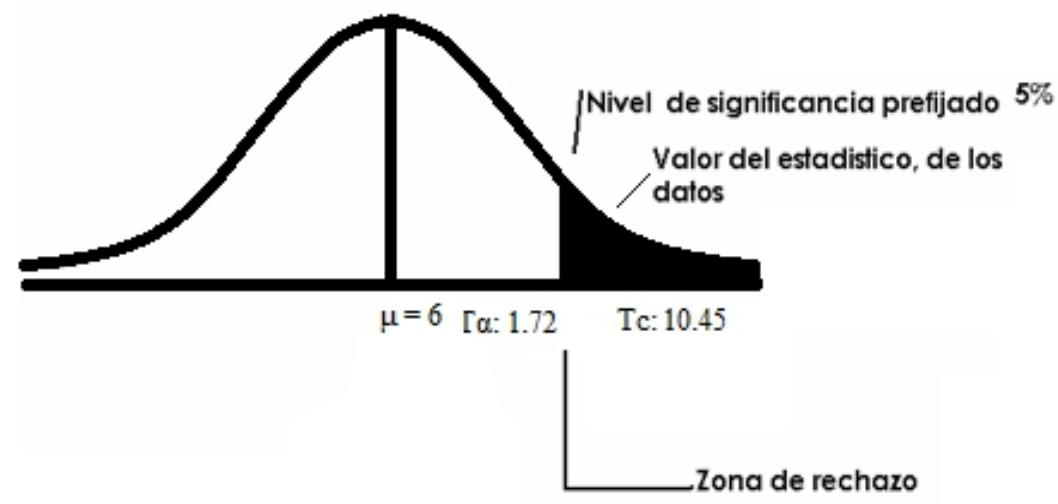

Fuente: Encuesta

Elaborado por: Msc. Marco Velasco

\section{Interpretación}

El estadístico calculado $t_{c}=10.45$ cae en la región de rechazo (se rechaza la hipótesis nula en favor de la hipótesis alternativa) por lo tanto existe suficiente evidencia estadística para afirmar: La selección y uso de aditamentos de protección aditiva (APA) en el programa de Conservación Auditiva para la prevención de alteraciones de los trabajadores ocupacionalmente expuestos a ruido de los departamentos de equipos pesado y turbina de la Empresa Pública de Hidrocarburos del Ecuador del Cantón Shushufindi brindan al trabajador un ambiente laboral sano

\section{Demostración de la Hipótesis $\mathbf{N}^{\circ} 5$}

Las evaluaciones audiométrica periódicas en el programa de Conservación Auditiva para la prevención de alteraciones de los trabajadores ocupacionalmente expuestos a ruido de los departamentos de equipos pesado y turbina de la Empresa Pública de Hidrocarburos del Ecuador del Cantón Shushufindi brindan al trabajador un ambiente laboral sano. 
Figura 5. Prueba de Hipótesis 5

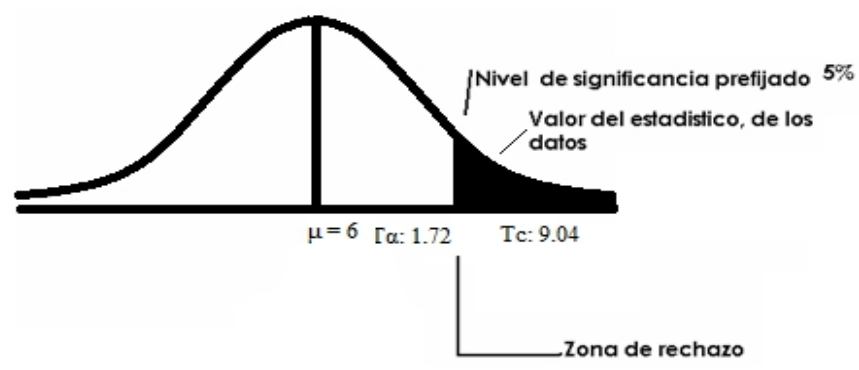

Fuente: Encuesta

Elaborado por: Msc. Marco Velasco

\section{Interpretación}

El estadístico calculado $t_{c}=9.048$ cae en la región de rechazo (se rechaza la hipótesis nula en favor de la hipótesis alternativa) por lo tanto existe suficiente evidencia estadística para afirmar: Las evaluaciones audiométrica periódicas en el programa de Conservación Auditiva para la prevención de alteraciones de los trabajadores ocupacionalmente expuestos a ruido de los departamentos de equipos pesado y turbina de la Empresa Pública de Hidrocarburos del Ecuador del Cantón Shushufindi brindan al trabajador un ambiente laboral sano.

\section{Conclusion}

El plan de conservación auditiva en los departamentos de equipo pesado y turbina, del campo Shushufindi permitió cumplir con las leyes y normativas legales vigentes ya que se evaluó, midió, y controlo el factor de riesgo en este caso el ruido, generando niveles de control acorde a la exposición.

Con la realización de mantenimiento y limpieza preventivo a las máquinas, evitando la propagación del ruido, y el uso obligatorios de los equipo protección personal dentro del programa de conservación auditiva permitió generar un ambiente sano, seguro y productivo.

La inducción inicial, las charlas médicas ocupacionales brindadas al trabajador permitieron en el programa de conservación auditiva, educar y capacitar sobre los efectos nocivos que genera el ruido en áreas expuestas.

El uso correcto del equipo de protección personal acorde a la exposición de ruido laboral permitió reducir los niveles de presión sonora y lograr estar dentro de los límites permisibles y la dosis de exposición en la 
prevención que el programa de conservación auditiva determinar cómo control en las áreas.

Las evaluaciones audiométricas lograron determinar los trabajadores expuestos y el grado de pérdida auditiva y las medidas de vigilancia médica a seguir aplicando el diagnostico de prevención terapéutica.

\section{References:}

1. CDC Office of Health and Safety. CDC Hearing Conservation Program. [Monograph on Internet]; 2004. [Cited 2005 Jun 10]. Available from:

2. http://www.cdc.gov/od/ohs/manual/hearing.htm

3. DIXON WW. Deterioro auditivo inducido por ruido. En: Paparella, M. Tratado de Otorrinolaringología. Tomo II. Cap. 35. La Habana: Edición Revolucionaria; 1982. p. 1772-88.

4. DUAN ML, Ulfendahl M, Ahlberg A, Pyykko I, Borg E. [Future cure of hearing disorders: Gene therapy and stem cell implantation are possible new therapeutic alternatives]. Lakartidningen. 2000;97(10):1106-12.

5. GILBERT Corzo A. Efectos de la exposición a ruido industrial. [Monografía en internet]. 2004. (Fecha de acceso 10 de junio de 2005). Disponible en:

6. http://www.medspain.com/colaboraciones/ruidoindustrial.htm

7. HERNANDO R. Salud ocupacional en Venezuela. [Monografía en Internet]. 1998. (Fecha de acceso 10 de junio de 2005). Disponible en:

8. http://members.tripod.com/RENDILES/OCUPACIONAL.html

9. MARTÍNEZ JA. Ruido y sordera: Sordera profesional por ruido. Salamanca: Ed. Graficesa; 1969.

10. Organización Panamericana de la Salud. Plan regional en salud de los trabajadores. [Monografía en Internet]. 2001.( Fecha de acceso 10 de junio 2005). Disponible en: http://www.who.int/entity/occupational_health/regions/en/oehamplan reg.pdf

11. Protocolos de diagnóstico y evaluación médica para enfermedades profesionales. Seguro complementario de trabajo de riesgo. DS. No 003-98-SA, Lima, 2004.

12. Rev Fac Med Unam. Hipoacusia inducida por ruido: un problema de salud y de conciencia pública. 2000 marzo-abril;43(2).

13. Trauma acústico o daño auditivo inducido por ruido (DAIR). Fundación Arauz-Instituto Oto-Rino-Laringológico. [Monografía en Internet], 2003 [Fecha de acceso 10 de junio de 2005]. Disponible en: http://www.sinfomed.org.ar/mains/publicaciones/traumaacus.htm 